\title{
Crecimiento Y Partición De Biomasa De Dos Cultivares De Maní (Arachis hypogaea L.) En Distintas Fechas De Siembra En Río Cuarto, Córdoba (Argentina)
}

Federico D. Morla, Dr. Oscar Giayetto, MSc.

Guillermo A. Cerioni, MSc.

Elena M. Fernandez, Dra.

Departamento de Producción Vegetal. Facultad de Agronomía y Veterinaria. Universidad Nacional de Río Cuarto. Río Cuarto - Córdoba - Argentina

doi: 10.19044/esj.2016.v12n30p334 URL:http://dx.doi.org/10.19044/esj.2016.v12n30p334

\begin{abstract}
Crop management practices like the choice of sowing date and of the cultivar modify and affect the environmental conditions of radiation and temperature in which peanut crop grows and develops. The aim of this study was to evaluate the effect that three contrasting sowing dates (SD) had on the growth and partition of biomass of two peanut cultivars of different cycle length; in the peanut-region of Rio Cuarto, Córdoba (Argentina). The experiment was conducted under field circumstances, without restrictions in water, nutrition or sanitary conditions during 2009/10 and 2010/11 growing season in the experimental field of the FAV-UNRC. On a Typical Haplustol soil with a clear light sandy texture. The Granoleico cultivars (runner type with cycle $>155$ days) and Utre cultivars (cross runner $x$ Spanish cycle of 130-140 days) were sown in early October, November and December (1st, 2nd and 3rd SD, respectively). During the growth cycle, environmental conditions were recorded, ecophysiological variables of development (phenology, thermal date) and growth (radiation interception, crop growth rate, biomass partitioning factor) of the crop were determined. At harvest, the yield components and market quality were determined, and the conditions to which the crop was exposed were analyzed. The phenological stages of peanut occurred in different environmental regimen due to variations in cycle length of the cultivars, sowing dates and the interaction between these variables. During the early SD, the duration of the cycle was longer, obtaining a greater amount of radiation as compared to the other SD. Both parameters, duration of the cycle and intercepted radiation (IPAR),
\end{abstract}


decreased with late sowing. By delaying the SD, the crop growth rate (CGR) is greater during the initial periods of the crop cycle; an anticipation in the moment of maximum CGR can be seen, changes that occur in response to increased temperature and incident radiation (PAR), reflected in the pattern of biomass production and partition to the different plant organs. The combination of the SD and the length cycle of the cultivars affected the location of the critical period of definition of the yield components and the amount of radiation obtained by the crop. Particularly, the weight of ripe pod and grains was affected, which decreased by delaying the SD, with similar behavior in both cultivars and years of study. As a result, the pod yield and market quality is reduced with the delay of sowing date in both cultivars.

Keywords: Peanut (Arachis hypogaea L.), Sowing Date, Radiation Use efficiency RUE, Crop Growth Rate CGR, Partition.

\section{Resumen}

Prácticas de manejo del cultivo como la elección de la fecha de siembra y del cultivar modifican las condiciones ambientales de radiación y temperatura en las que crece y se desarrolla el cultivo de maní, afectándolo. El objetivo de este trabajo fue evaluar el efecto de tres fechas de siembra (FS) contrastantes, sobre el crecimiento y partición biomasa de dos cultivares de maní de diferentes longitud de ciclo en la región manisera de Río Cuarto, provincia de Córdoba (Argentina). El experimento se llevó a cabo en condiciones de campo y sin limitantes hídricas, nutricionales ni sanitarias, durante los ciclos 2009/10 y 2010/11, en el Campo de Docencia y Experimentación de la FAV-UNRC. Sobre un suelo Haplustol típico de textura franca arenosa fina. Se sembraron los cultivares Granoleico (tipo runner de ciclo > 155 días) y Utre (cruzamiento runner x Español de ciclo de 130 a 140 días), a principios de octubre, noviembre y diciembre $\left(1^{\circ}, 2^{\circ}\right.$ y $3^{\circ}$ fecha de siembra, respectivamente). Durante el ciclo de crecimiento se registraron las condiciones ambientales y se determinaron variables ecofisiológicas del desarrollo (fenología, tiempo térmico) y crecimiento (radiación interceptada, tasa de crecimiento del cultivo, factor de partición de biomasa) del cultivo. A cosecha, se determinaron los componentes del rendimiento y la calidad comercial y se analizaron las condiciones a las que estuvo expuesto el cultivo. Las etapas fenológicas del cultivo de maní ocurrieron en distintos regímenes ambientales debido a variaciones en la longitud del ciclo de los cultivares, la fecha de siembra y la interacción entre estas variables. En la FS temprana la duración del ciclo fue mayor, capturando más cantidad de radiación respecto a las otras fechas de siembra. Ambos parámetros, duración del ciclo y radiación interceptada (RFAi), disminuyeron con el atraso de la siembra. Al retrasar la FS la TCC es mayor 
durante los periodos iniciales del ciclo del cultivo, observándose un adelantamiento del momento de TCC máxima, cambios que suceden en respuesta a una mayor temperatura y radiación incidente (RFAinc), que se refleja en el patrón de producción de biomasa y su partición a los diferentes órganos de la planta. La combinación entre fecha de siembra y longitud de ciclo de los cultivares afectaron la ubicación del período crítico de definición de los componentes del rendimiento y la cantidad de energía radiante capturada por el cultivo durante los mismos. En particular, resultó afectado el peso de frutos maduros y granos, que disminuyeron al atrasar la fecha de siembra, con un comportamiento similar en ambos cultivares y años de estudio. En consecuencia, el rendimiento de frutos y la calidad comercial del producto se redujeron con el atraso de la fecha de siembra en ambos cultivares.

Palabras clave: Maní (Arachis hypogaea L.), Fecha de Siembra, eficiencia de uso de la radiación, Tasa de crecimiento, Partición.

\section{Introducción}

El maní (Arachis hypogaea L.) es uno de los cultivos oleaginosos más importante en regiones tropicales y subtropicales del mundo. Argentina es el principal exportador mundial de maní, y el $96 \%$ de la superficie cultivada se concentra en el centro-sur de la provincia de Córdoba. Esta región, debido a la latitud donde se ubica (entre $31^{\circ} 30^{\prime}$ y $35^{\circ}$ de latitud sur), presenta escenarios ambientales sub-óptimos de temperatura y radiación para el crecimiento y desarrollo del cultivo. Factores que, además, varían mucho durante la estación de crecimiento del cultivo (Padmalatha et al., 2006; Haro et al., 2007a), con la longitud del ciclo del cultivar (Bell et al., 1991; Nanja Reddy et al., 1995), la fecha de siembra (Bell, 1986; Canavar y Kaynak 2010) y sus interacciones (Nanja Reddy et al., 1995; Chandrika et al., 2008; Prathima et al., 2012).

Estos escenarios sub-óptimos están configurados por la extensa longitud del ciclo de los cultivares utilizados (>150 días) (Gamba et al., 2014) y el alto requerimiento de temperatura del maní con óptimos entre 25 a $30^{\circ} \mathrm{C}$ (Kumar et al., 2012). A ello se suma la frecuente ocurrencia de precipitaciones en el otoño y un descenso concomitante de la radiación incidente y la temperatura, que afectan negativamente el llenado de frutos (Giayetto et al., 2006).

Con la elección de la fecha de siembra (FS) y el ciclo del cultivar para cada ambiente se procura configurar el mejor escenario ambiental para las etapas claves del crecimiento del cultivo. Determinación que se hace aún más compleja a causa de las interacciones (FS x año, FS x cultivar y FS x localidad) que se han constatado (Nanja Reddy et al., 1995). 
La siembra de maní en esta región se realiza en la primavera, recomendándose comenzar cuando el suelo tiene, como mínimo, $18^{\circ} \mathrm{C}$ a la profundidad de siembra durante tres días consecutivos (Pedelini, 2014). Según registros meteorológicos históricos, esa condición se produce en la tercera década del mes de octubre (Giayetto et al., 2006). Sin embargo, últimamente existe una tendencia a adelantar la fecha de siembra hacia la primera década de octubre por razones operativas (siembras de grandes superficies, necesidad de coordinar las operaciones de arrancado y descapotado); siempre que la humedad del suelo sea adecuada para la imbibición de la semilla y la temperatura no inferior a $12^{\circ} \mathrm{C}$ (Giayetto et al., 2006; Pedelini, 2014).

En la región central de Córdoba y durante dos años, Giambastiani (2000) evaluó fechas de siembra temprana ( $1^{\circ}$ década de octubre), intermedia ( $1^{\circ}$ década de noviembre) y tardía ( $1^{\circ}$ década de diciembre) y obtuvo menor rendimiento en la siembra tardía respecto a la temprana e intermedia que no se diferenciaron entre sí.

Haro et al. (2007a) evaluaron dos fechas de siembra, 27/10/06 (temprana) y 6/12/06 (tardía) en Manfredi (Córdoba) con un cultivar tipo runner. En la fecha temprana el cultivo recibió menos radiación y temperatura durante las etapas iniciales del ciclo y aumentaron desde el comienzo de llenado de granos hasta la cosecha (R5 a R8) (Boote, 1982). Además, la duración del ciclo, producción de biomasa, rendimiento y calidad confitería fueron mayores que en la siembra tardía.

En otro estudio similar, Haro et al. (2007b) involucrando otros ambientes y dos cultivares tipo runner (ASEM 485 y Florman INTA), corroboraron la relación estrecha entre número de granos y rendimiento; componente que, a su vez, depende de las tasas de crecimiento del cultivo (TCC) y de los frutos (TCF) durante el período crítico de su determinación (R3-R6,5). Además, ambas tasas de crecimiento se relacionaron con la radiación interceptada. Las distintas fechas de siembra afectaron la dinámica de la radiación respecto de las etapas fenológicas del cultivo, siendo las siembras tardías (21/12) las que recibieron menos radiación durante el período reproductivo. Estos autores, también encontraron en esas siembras que disminuyó la eficiencia de uso de la radiación (EUR) debido a un cambio en la relación fuente-destino atribuido a un menor número de frutos.

En otras condiciones ambientales, Caliskan et al. (2008) mostraron que las siembras de maní en fechas intermedias tuvieron mayor rendimiento que las siembras muy tempranas o más tardías. En esas fechas intermedias el cultivo estuvo expuesto a un régimen de temperatura adecuado y recibió más radiación solar.

Sobre la misma base de datos de este trabajo, encontramos que el rendimiento de frutos y la calidad comercial (rendimiento confitería) se 
redujeron lineal y negativamente con el atraso de la fecha de siembra en los cultivares evaluados (Giayetto et al., 2012). Sin embargo, no se ha descripto el efecto de diferentes fechas de siembra sobre las variables ecofisiológicas que determinan el crecimiento y la partición de biomasa en el cultivo de maní.

El objetivo de este trabajo fue evaluar el efecto de la fecha de siembra temprana, intermedia y tardía sobre el crecimiento y partición biomasa de dos cultivares de maní de diferente longitud de ciclo en la región manisera de Río Cuarto, provincia de Córdoba (Argentina).

\section{Materiales y Métodos}

Los ensayos experimentales se realizaron en dos ciclos agrícolas (2009/10 y 2010/11), en el Campo de Docencia y Experimentación de la Facultad de Agronomía y Veterinaria de la Universidad Nacional de Río

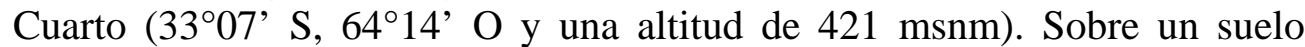
Haplustol típico (Taxonomía de Suelos del USDA) de relieve normal, profundo, bien drenado, de bajo contenido de materia orgánica y textura franca arenosa muy fina. El cultivo se condujo sin restricciones hídricas, para lo cual se aplicaron periódicamente riegos complementarios mediante un sistema por goteo. Las malezas, insectos y enfermedades fueron adecuadamente controlados. El diseño experimental utilizado fue de bloques completos al azar dispuestos en un arreglo factorial de parcelas divididas y tres repeticiones espaciales por tratamiento. El factor principal fue la fecha de siembra con 3 niveles: Temprana ( $\left.1^{\circ} \mathrm{FS}\right), 08$ de octubre en ambos ciclos), Media ( $2^{\circ} \mathrm{FS}$ ), 10 de noviembre en ambos ciclos) y Tardía ( $3^{\circ} \mathrm{FS}$ ), 09 y 10 de diciembre de 2009 y 2010, respectivamente). El factor secundario fue el cultivar, utilizándose Utre (de 130-140 días de ciclo, porte semierecto y patrón de ramificación hasta $n+2$ ) y Granoleico (de ciclo largo -alrededor de 160 días-, porte rastrero y ramificación hasta $n+3)$. Las siembras se realizaron manualmente en surcos distanciados a 0,70 m y a $0,08 \mathrm{~m}$ entre plantas. Las parcelas experimentales constaban de 9 surcos de $12 \mathrm{~m}$ de largo. Se colocaron dos semillas por golpe a una profundidad de 3-5 cm. Luego de producida la emergencia, se efectuó un raleo manual dejando una planta por golpe lo que correspondió a una densidad de 17,8 plantas $\mathrm{m}^{-2}$ en ambos cultivares. Los registros diarios de las variables meteorológicas se obtuvieron de una estación meteorológica automática situada en las proximidades de la parcela. El seguimiento de la fenología del cultivo en las distintas fechas de siembra se realizó in situ, mediante el registro de la fecha de ocurrencia en días calendario de las principales etapas fenológicas del cultivo (Boote, 1982). Esas etapas fueron, además, referenciadas cronológicamente en grados día $\left({ }^{\circ} \mathrm{Cd}\right)$, calculados con la ecuación lineal de tiempo térmico (TT) empleando una temperatura base $(\mathrm{Tb})$ de $10,3^{\circ} \mathrm{C}$ (Kumar et al., 2012). 
Durante el periodo reproductivo del cultivo, desde floración (etapa R1) hasta R8 (madurez de arrancado), se tomaron muestras de 10 plantas en competencia perfecta a intervalos regulares de 10 a 13 días. En cada muestreo, las plantas fueron separadas en sus órganos componentes (tallo/ramas, hojas, frutos y semillas) y secados en estufa hasta peso constante. La biomasa reproductiva (frutos) fue corregida por un coeficiente de ajuste energético de 1,65 (Duncan et al., 1978). Con los datos de biomasa se construyeron las curvas de crecimiento acumulado, se calcularon las tasas de crecimiento de frutos (TCF) y del cultivo (TCC) y, de la relación entre ellas, se obtuvo el factor de partición (TCF/TCC). En cada fecha de muestreo para la medición de biomasa, se registró el porcentaje de intercepción de la radiación (IR) y la radiación fotosintéticamente activa interceptada (RFAi) mediante LI-191SA (Line Quantum Sensor LICOR, Lincoln, NE, USA). Para determinar el tiempo que el cultivo tarda en interceptar el 95\% de la radiación incidente, se graficó la fracción interceptada en función del tiempo térmico desde la siembra para cada cultivar y fecha de siembra. La eficiencia de uso de la radiación (EUR) del cultivo fue calculada mediante regresiones lineales entre los valores de materia seca producida y RFAi acumulados durante el ciclo del maní. Al momento de cosecha (etapa R8) se tomaron muestras de $1 \mathrm{~m}^{2}$ por cultivar, fecha de siembra y repetición para evaluar: número y peso de frutos maduros e inmaduros, número de granos por vaina, peso medio de un fruto, peso de semillas y peso de la parte vegetativa. Todas las variables fueron sometidas a análisis estadísticos para detectar diferencias significativas mediante ANAVA y comparaciones múltiples mediante test de mínima diferencia significativa LSD de Fisher $(\alpha \leq 0,05)$. También se realizaron pruebas de homogeneidad de pendientes y correlaciones entre las variables analizadas, con el programa estadístico Infostat versión 2014 y el programa GraphPad Prism versión 5.00 para Windows, (GraphPad Software, San Diego California USA).

\section{Resultados y Discusión}

Fenología y temperatura

El atraso de la fecha de siembra produjo un acortamiento del ciclo total (S a R8) de ambos cultivares en los dos años (Cuadro 1), cuya duración promedio (ciclos y cultivares) fue de 159,152 y 137 días para la $1^{\circ}, 2^{\circ}$ y $3^{\circ}$ FS, respectivamente. El 65\% de ese acortamiento se produjo en el periodo vegetativo (S-R1) y el resto entre las etapas R1-R5 y R5-R8, con porcentajes de reducción similares en ambas. Estos resultados son consistentes con la literatura. Haro et al. (2007a) describieron una reducción de 14 días en una siembra de fines de noviembre respecto a otra de mediados de octubre, realizadas en Manfredi (Córdoba) con un cultivar tipo runner (Florman INTA), de características similares a Granoleico. Canavar y Kaynak (2010) 
también encontraron diferencias en la duración del ciclo del cultivo según fechas de siembra, con valores de 169, 157, 148 y 143 días para un cultivar tipo runner sembrado a intervalos de 14 días entre sí.

El acortamiento del ciclo coincidió con el aumento progresivo de la temperatura media diaria asociado al avance de la estación estival. Entre $\mathrm{S}$ y $\mathrm{R} 1$, la temperatura media aumentó $1,9{ }^{\circ} \mathrm{C}$ (Utre 2009/10) y 4,9 ${ }^{\circ} \mathrm{C}$ (Utre 2010/11) como valores extremos entre la $1^{\circ}$ y $3^{\circ} \mathrm{FS}$ (Cuadro 2), con valores intermedios para la $2^{\circ} \mathrm{FS}$. El aumento de la temperatura media, aceleró la tasa de desarrollo del cultivo que resultó en una duración promedio de 14 días menos en la $3^{\circ} \mathrm{FS}$ respecto de la $1^{\circ} \mathrm{FS}$. Este comportamiento fue documentado por Bell et al. (1991); Haro et al. (2007b); Chandrika et al. (2008); Prathima et al. (2012). Contrariamente, la temperatura media de la etapa R5-R8 disminuyó entre la $1^{\circ}$ y $3^{\circ} \mathrm{FS}$; aunque la duración de esa etapa también se redujo, cambio que podría indicar un efecto del fotoperiodo sobre el desarrollo del cultivo. Diferencias de temperatura media entre cultivares sólo se encontraron en la etapa final del ciclo (R5-R8). El patrón de temperatura creciente en las etapas iniciales y decreciente en las finales del ciclo del cultivo, determinó que no se manifestaran diferencias entre las FS al considerar el ciclo completo de los cultivares. Así, la temperatura media del ciclo total varió entre 20,3 y $21,9{ }^{\circ} \mathrm{C}$ con un promedio de $21,1{ }^{\circ} \mathrm{C}$.

El ciclo experimental 2009/10 presentó temperaturas medias mayores durante el ciclo (S-R8) de los cultivares respecto a las registradas en 2010/11 (21,5 vs $20,6{ }^{\circ} \mathrm{C}$, respectivamente). Además, Utre por su ciclo más corto, estuvo expuesto a temperaturas medias diarias mayores $\left(20,3{ }^{\circ} \mathrm{C}\right)$ que las experimentadas por Granoleico $\left(19,2^{\circ} \mathrm{C}\right)$, cuyo ciclo fue unos 30 días más largo.

\section{Fenología y radiación}

Los cambios debidos a la fecha de siembra en la localización temporal del ciclo de los cultivares (S-R8), determinaron que los sembrados en la $1^{\circ}$ y $2^{\circ} \mathrm{FS}$ recibieran, en promedio, mayor cantidad de radiación solar (1961 y $1820 \mathrm{MJ} \mathrm{m}^{-2}$, respectivamente), que en la $3^{\circ} \mathrm{FS}$ (1319 $\mathrm{MJ} \mathrm{m}^{-2}$ ) (Cuadro 2). Estas diferencias en cantidad de RFAinc estuvieron a favor de la $1^{\circ}$ FS en casi todas las etapas fenológicas evaluadas. También hubo diferencias entre cultivares donde Granoleico, por su mayor longitud de ciclo, recibió más RFAinc que Utre (1828 y $1738 \mathrm{MJ} \mathrm{m}^{-2}$, respectivamente). Por su parte, el ciclo experimental 2010/11 tuvo mayores valores de RFAinc (1957 y $1858 \mathrm{MJ} \mathrm{m}^{-2}$ para Granoleico y Utre, respectivamente) que el 2009/10 (1699 y $1619 \mathrm{MJ} \mathrm{m}^{-2}$ para Granoleico y Utre, respectivamente). 
Intercepción de la radiación

Los cultivares utilizados en este estudio superaron el 95\% de intercepción de la RFAinc en las 3 fechas de siembra y los dos ciclos experimentales evaluados (Figura 1). En Granoleico, ese momento sucedió, en promedio, alrededor de la formación de frutos (etapa R4) (entre 938 y $1172{ }^{\circ} \mathrm{Cd}$ en $2009 / 10$ y entre 1058 y $1082{ }^{\circ} \mathrm{Cd}$ en $2010 / 11$ ); y en Utre se produjo en R5-R6 (entre 1067 y $1298{ }^{\circ} \mathrm{Cd}$ en 2009/10 y entre 1019 y 1285 ${ }^{\circ} \mathrm{Cd}$ en 2010/11).

En el ciclo experimental 2009/10, se observó el mismo patrón temporal de llegada de ambos cultivares al 95\% de intercepción de la radiación. En primer lugar, la $1^{\circ} \mathrm{FS}$ seguida por la $3^{\circ}$ y $2^{\circ} \mathrm{FS}$ con poca diferencia entre ellas. En cambio, en el ciclo 2010/11 la $2^{\circ}$ FS alcanzó más rápidamente el 95\% de intercepción en ambos cultivares, con diferencias entre ellos en el momento de ocurrencia de esa condición. El segmento del ciclo del cultivo (expresado en ${ }^{\circ} \mathrm{Cd}$ ) con una intercepción de RFA superior al 95\% fue diferente entre cultivares y fechas de siembra. En Utre, ese período fue menor (321 y $469^{\circ} \mathrm{Cd}$ ). En general, la $2^{\circ} \mathrm{FS}$ presentó la mayor duración (469 y $381{ }^{\circ} \mathrm{Cd}$ ) seguida por la $1^{\circ} \mathrm{FS}$ (en 2009/10, $360{ }^{\circ} \mathrm{Cd}$ ) y por la $3^{\circ} \mathrm{FS}$ (en 2010/11, $335{ }^{\circ} \mathrm{Cd}$ ). En Granoleico, la duración de ese segmento fue mayor (347 y $787^{\circ} \mathrm{Cd}$ ), y mostró una tendencia decreciente según la fecha de siembra de 787 a $633{ }^{\circ} \mathrm{Cd}$ en la $1^{\circ} \mathrm{FS}$, de 535 a $546{ }^{\circ} \mathrm{Cd}$ en la $2^{\circ} \mathrm{FS}$ y de 353 a $347{ }^{\circ} \mathrm{Cd}$ en la $3^{\circ} \mathrm{FS}$, en 2009/10 y 2010/11, respectivamente. Diferencias en la intercepción de la radiación entre cultivares de distinto porte son descriptas por Lanier et al. (2004), quienes señalan que los cultivares de porte erecto son más beneficiados al acortar la distancia entre surcos. En este sentido, Huyghe (1998) muestra que el patrón de ramificación tiene gran importancia en los cambios de la intercepción de la RFA, sobre todo cuando la ramificación tiene lugar al comienzo del ciclo, debido a la variación en el número de hojas por planta y la modificación de su distribución tridimensional.

Considerando la cantidad de RFAinc (Cuadro 2) y la eficiencia de su intercepción (Figura 1), Utre interceptó $1132 \mathrm{MJ} \mathrm{m}^{-2}$ durante la $2^{\circ} \mathrm{FS}$ del ciclo 2010/11 como valor máximo. En promedio, esa fecha de siembra logró la mayor cantidad de RFAi (1024 MJ m²), seguida por la $1^{\circ}$ y $3^{\circ} \mathrm{FS}$ (con 997 y $980 \mathrm{MJ} \mathrm{m}^{-2}$, respectivamente). En Granoleico, la mayor cantidad de RFAi fue en la $1^{\circ} \mathrm{FS}\left(1123 \mathrm{MJ} \mathrm{m}^{-2}\right)$-con un registro máximo de $1244 \mathrm{MJ} \mathrm{m}^{-2}$ durante el ciclo 2010/11- disminuyendo con el atraso de la fecha de siembra entre 7 y $8 \%$ en la $2^{\circ} \mathrm{FS}$ y entre 21 y $29 \%$ en la $3^{\circ} \mathrm{FS}$. Valores similares fueron encontrados por Haro et al. (2007a). Sin embargo, en el cultivar Utre la mayor radiación acumulada se registró en la $2^{\circ} \mathrm{FS}$ con una disminución del orden del $3 \%$ en una FS más temprana y de $5 \%$ en otra más tardía. Este 
tipo de respuesta ha sido descripta, por ejemplo, en variedades de soja de ciclos cortos (Kane y Grabau, 1992).

Las diferencias entre cultivares en la intercepción de la radiación se deben a la longitud del ciclo y al porte (grado de ramificación) de los mismos. El mayor tiempo que necesitó Utre para alcanzar el 95\% de intercepción de la RFAinc (Figura 1), fue a causa de su porte erecto y menor grado de ramificación. Similares resultados fueron encontrados a nivel local donde se describe una mayor intercepción de la radiación incidente en un cultivar de tipo rastrero (Florman INTA) respecto a otro de porte semierecto (Manfredi 393 INTA) (Collino et al., 2001). Por su parte, Granoleico estuvo más tiempo (31\%) interceptando un alto porcentaje de RFAinc (95\% o superior) que Utre, evidenciando la diferencia en la longitud del ciclo del cultivo entre estos tipos botánicos de maní. En este sentido, diferentes autores encontraron, en ensayos con distintas fechas de siembra (Nanja Reddy et al., 1995) y tipos botánicos de maní (Haro et al., 2013), que los rendimientos altos se relacionaron positivamente con la duración del periodo reproductivo.

\section{Eficiencia de uso de la radiación}

Los valores promedio de EUR encontrados en este estudio variaron entre 1,54 - 2,23 y entre 1,71 - 2,68 g MJ ${ }^{-1}$ (RFAi) en Utre y Granoleico, respectivamente; siendo, en todos los casos, superiores en Granoleico. El test de homogeneidad de pendientes mostró que la EUR de todas las fechas de siembra no presentaron diferencias significativas en Utre 2009/10 $(p=0,31) \mathrm{y}$ 2010/11 $(p=0,33)$ ni en Granoleico 2009/10 ( $p=0,36)$ y 2010/11 ( $p=0,26)$. Por ello, se calculó una sola pendiente para el conjunto de datos (Figura 2).

Por otro lado, el ciclo experimental 2010/11 presentó, en ambos cultivares, mayores valores de EUR (2,03 y 2,56 g $\mathrm{MJ}^{-1}$ para Utre y Granoleico, respectivamente) que los registrados en 2009/10 (1,67 g MJ ${ }^{-1}$ en Utre y 1,79 $\mathrm{g} \mathrm{MJ}^{-1}$ en Granoleico). En consecuencia, las variaciones en la biomasa total producida por el cultivo estuvieron más relacionadas con cambios en la cantidad de RFA interceptada que en la EUR.

A diferencia de lo descripto por Haro et al. (2007b), en este trabajo no se produjo una diminución de la EUR en la etapa final del ciclo del cultivo en las fechas de siembra tardías. Estos autores encontraron una correlación entre la EUA y el número de destinos fijados (granos). Pero, en este trabajo no se observó una disminución del número de frutos cosechados en respuesta a las fechas de siembra (Cuadro 3). Quizás debido a que, en contraste con lo encontrado por Haro et al. (2007b), en este estudio no hubo diferencias en la temperatura media y RFAinc a la que estuvo expuesto el cultivo en la etapa de definición de ese componente del rendimiento (R1 a R5) (Cuadro 2). En concordancia, Kiniry et al. (2005) señalan que en maní la 
EUR es relativamente estable para un amplio rango de condiciones ambientales y de manejo con valores entre 1,71 y 2,32 g $\mathrm{MJ}^{-1}$ de RFA interceptada

\section{Crecimiento y Partición}

La dinámica temporal de la TCC mostró que los valores máximos ocurrieron antes en la $3^{\circ} \mathrm{FS}$ de ambos cultivares y ciclos experimentales (Figura 3). En Utre, y como promedio de ambos ciclos experimentales, esos valores ocurrieron a los 1386,1287 y $1085{ }^{\circ} \mathrm{Cd}$ en la $1^{\circ}, 2^{\circ}$ y $3^{\circ} \mathrm{FS}$, respectivamente. Por su parte, en Granoleico los valores máximos de TCC tuvieron una ocurrencia temporal similar registrándose a los 1481, 1225 y $1084{ }^{\circ} \mathrm{Cd}$ en la $1^{\circ}, 2^{\circ}$ y $3^{\circ} \mathrm{FS}$, respectivamente. Los mayores valores de TCC de Utre se produjeron en la $3^{\circ} \mathrm{FS}$ del ciclo 2010/11 $\left(2,88 \mathrm{~g} \mathrm{~m}^{-2}{ }^{\circ} \mathrm{Cd}^{-1}\right)$, seguida por la $2^{\circ} \mathrm{FS}$ del mismo ciclo $\left(2,80 \mathrm{~g} \mathrm{~m}^{-2}{ }^{\circ} \mathrm{Cd}^{-1}\right)$ y luego la $3^{\circ} \mathrm{FS}$ del ciclo $2009 / 10\left(2,18 \mathrm{~g} \mathrm{~m}^{-2}{ }^{\circ} \mathrm{Cd}^{-1}\right)$, por último se ubicaron la $1^{\circ} \mathrm{FS}$ del ciclo

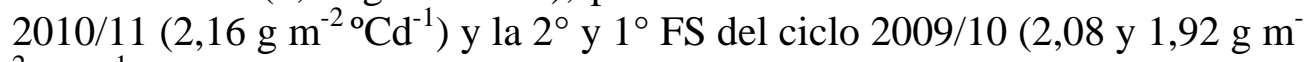
${ }^{2}{ }^{\circ} \mathrm{Cd}^{-1}$, respectivamente). Este cultivar mostró mayores diferencias entre ciclos experimentales; mientras que Granoleico no tuvo grandes cambios entre ciclos. Los mayores valores se dieron en la $2^{\circ}$ FS del ciclo 2010/11 $\left(2,72 \mathrm{~g} \mathrm{~m}^{-2}{ }^{\circ} \mathrm{Cd}^{-1}\right)$, en la $1^{\circ}$ y $3^{\circ} \mathrm{FS}$ del ciclo $2009 / 10\left(2,71\right.$ y $2,51 \mathrm{~g} \mathrm{~m}^{-2}{ }^{\circ} \mathrm{Cd}^{-1}$, respectivamente) seguidos por la $2^{\circ} \mathrm{FS}$ del mismo ciclo $\left(2,41 \mathrm{~g} \mathrm{~m}^{-2}{ }^{\circ} \mathrm{Cd}^{-1}\right) \mathrm{y}$ la $1^{\circ}$ y $3^{\circ} \mathrm{FS}$ del ciclo 2010/11 (2,37 y 1,69 $\mathrm{g} \mathrm{m}^{-2}{ }^{\circ} \mathrm{Cd}^{-1}$, respectivamente).

En general, la dinámica temporal de la TCC siguió la marcha de la temperatura. Al analizar las relaciones entre TCC (Figura 3) y variables ambientales (Cuadro 2), se observó que la temperatura entre R1 y R5 fue la variable ambiental que más se correlacionó con el tiempo en alcanzar la TCC máxima $(\mathrm{r}=0,60 ; p=0,02)$. En cambio, en la determinación de los valores máximos de TCC las variables ambientales que más influyeron fueron la radiación incidente $(\mathrm{r}=0,56 ; p=0,04)$ y la temperatura $(\mathrm{r}=0,65 ; p=0,04)$ durante el periodo S-R1. En este sentido, otros autores (Collino et al., 2001; Haro et al., 2007b) señalan que el crecimiento, principalmente en las últimas etapas del ciclo del cultivo, responde altamente a la actividad de los destinos de fotoasimilados (frutos). Además, se han reportado mayores tasas de fotosíntesis neta en diferentes cultivares de maní bajo temperaturas de $35 / 30^{\circ} \mathrm{C}$ (día/noche) comparadas con tratamientos a temperaturas menores (25/20 ${ }^{\circ} \mathrm{C}$ día/noche) (Kumar et al., 2012).

En las siembras tardías, que crecieron con mayores temperaturas iniciales, el cultivo acumuló relativamente la mayor cantidad de biomasa vegetativa en ambos cultivares. Un indicador indirecto fue el menor tiempo transcurrido en alcanzar el 95\% de intercepción de la radiación; mientras que la partición de asimilados entre hojas y tallos dentro de la planta no se vio afectada por la fecha de siembra (datos no mostrados). 
Las diferencias en la partición inicial (R3) y final (R7-R8) a frutos entre las FS también pueden ser explicadas por la variación en la temperatura. El adelantamiento temporal de la partición a frutos observado principalmente en la $3^{\circ} \mathrm{FS}$ de ambos cultivares y ciclos experimentales (Figura 4), se relacionó positivamente con las mayores temperaturas que experimentó el cultivo durante los periodos S-R1 ( $\mathrm{r}=0,69 ; p=0,01)$. Así mismo, los mayores valores de partición a fruto se produjeron en la $1^{\circ}$ y $2^{\circ}$ FS de Granoleico (1,16 y 1,35, respectivamente) y de Utre (1,14 y 0,97, respectivamente) respecto a la $3^{\circ} \mathrm{FS}(1,07$ y 0,84 para Granoleico y Utre, respectivamente). Además, Granoleico (con 1,19 de partición promedio a frutos) superó a Utre $(0,98)$; mostrando, a su vez, una alta correlación con la temperatura de la etapa R5-R8 de ambos cultivares $(\mathrm{r}=0,57 ; p=0,02)$. Estos resultados son similares a los obtenidos por Bell (1986), quien encontró que las mayores particiones a frutos se obtenían con las mayores temperaturas. En ese sentido, Ong (1984) describió que la mayor partición a frutos expresada como la relación entre biomasa de frutos y la biomasa aérea- fue a los $22^{\circ} \mathrm{C}$ (valor similar a los del periodo R5-R8 en las fechas de siembras tempranas), y que la partición a frutos decrecía a medida que la temperatura media del aire se incrementaba o disminuía.

La variación en la partición encontrada en este trabajo, también se puede observar a través del índice de cosecha (Cuadro 3), que disminuyó notablemente en las siembras tardías, $(0,58$ y 0,62$)$ en las dos primeras fechas de siembra de los ciclos 2009/10 y 2010/11, disminuyendo significativamente $14 \%(p=0,0302)$ y $15 \%(p=0,0036)$ en la $3^{\circ}$ FS de los ciclos 2009/10 y 2010/11, respectivamente. Esta respuesta está documentada en numerosos trabajos (Bell et al., 1991; Padmalatha et al., 2006; Caliskan et al., 2008 en maní; y Cirilo y Andrade, 1994, en maíz). Esto podría deberse, además, a las condiciones ambientales más desfavorables (Haro et al., 2007b), a la mayor superposición de los estadios vegetativo y reproductivo en siembras tardías, por lo que la formación de tallos y hojas en crecimiento activo compiten por los asimilados con los procesos de formación de órganos reproductivos que definen el rendimiento y así disminuir el índice de cosecha (Padmalatha et al., 2006).

\section{Rendimiento}

Los resultados obtenidos en el presente estudio indican que el rendimiento fue significativamente influenciado por la fecha de siembra en ambos ciclos experimentales (Cuadro 3). El retraso en la siembra desplaza el periodo reproductivo del cultivo hacia condiciones declinantes de radiación y temperatura que inciden negativamente en los procesos involucrados en la determinación del rendimiento (Cirilo y Andrade, 1994; Kumar et al., 2012). Retrasar la fecha de siembra en este trabajo produjo una disminución 
significativa del peso individual del fruto, al igual que lo hallado por Chandrika et al. (2008) y Prathima et al. (2012); pero, contrariamente a lo señalado por otros autores (Haro et al., 2007b), no se detectaron cambios significativos en el número de frutos maduros por planta.

Granoleico superó significativamente a Utre en el número y el peso de frutos maduros y de granos por planta; mientras que el peso individual del fruto no se diferenció entre cultivares. La diferencia en el rendimiento entre los cultivares se debió a las características propias de los tipos runner y Español, como lo describen Haro et al. (2013). Estos autores, encontraron que las principales diferencias en el rendimiento potencial de los cultivares sembrados en Argentina en los últimos 60 años, se debieron a la introducción de los materiales runner de porte rastrero a mediados de la década de 1970 .

Además, en el presente trabajo y en coincidencia con Padmalatha et al. (2006) y Caliskan et al. (2008) se relacionó el aumento significativo del rendimiento, con la mayor duración del ciclo del cultivo.

La disminución del rendimiento confitería en respuesta a la FS, ajustó a un modelo lineal con $0,30 \%\left(\mathrm{R}^{2}=0,99\right)$ y $0,08 \%\left(\mathrm{R}^{2}=0,98\right)$ de caída para Granoleico y Utre, respectivamente (ciclo 2009/10); y de 0,58\% $\left(\mathrm{R}^{2}=0,93\right.$ ) por cada día de retraso respecto a la $1^{\circ}$ FS para ambos cultivares en 2010/11. Un resultado similar fue obtenido por Haro et al. (2007b), quienes encontraron en la siembra temprana el mayor porcentaje de la fracción maní confitería y mayores tasas de crecimiento del cultivo y de los frutos.

Para más información sobre la disminución del rendimiento y la calidad comercial del cultivo ante retrasos en la fecha de siembra véase Giayetto et al. (2012).

\section{Conclusión}

Las combinaciones de fechas de siembra (3), ciclos experimentales (2) y longitud de ciclo de los cultivares (2), configuraron diferentes escenarios de temperatura y radiación a los que se expuso el cultivo de maní durante su estación de crecimiento. En la $1^{\circ} \mathrm{FS}$ (primera década de octubre) en la región de Río Cuarto (Córdoba), la duración del ciclo de los cultivares de maní fue mayor y, por ello, capturaron más cantidad de radiación respecto a las otras fechas de siembra. Ambos parámetros, duración del ciclo y RFAi, disminuyeron con el atraso de la siembra (comienzos de noviembre y diciembre) debido a la respuesta inversa del desarrollo a la temperatura, particularmente notoria en la $3^{\circ} \mathrm{FS}$. Al retrasar la FS, la TCC es mayor durante los estadios iniciales del cultivo, observándose una anticipación del momento de TCC máxima; cambios que ocurren en respuesta a una mayor temperatura y RFAinc, y que se reflejan en el patrón de producción de biomasa y su partición a los diferentes órganos de la planta. Así, el índice de cosecha también disminuyó con el atraso de la fecha de siembra, 
particularmente en la más tardía (comienzos de diciembre). La EUR no se modificó con las diferentes FS, pero varió entre cultivares y ciclos experimentales. La combinación de fecha de siembra y duración del ciclo de los cultivares, modificó la ubicación del período crítico de definición de los componentes del rendimiento, y la cantidad de radiación capturada por el cultivo durante los mismos. En particular, resultó afectado negativamente el peso de frutos maduros y de granos, que disminuyeron al atrasar la fecha de siembra, con un comportamiento similar en ambos cultivares y años de estudio. En consecuencia, el rendimiento de frutos y la calidad comercial del producto se redujeron con el atraso de la fecha de siembra en ambos cultivares.

\section{References:}

1. Bell M.J., R. Shorter \& R. Mayer. (1991). Cultivar and environmental effects on growth and development of peanuts (Arachis hypogaea L.). II. Reproductive development. Field Crops Research, 27(1-2): 35-49.

2. Bell, M. (1986). Effect of sowing date on growth and development of irrigated peanuts (Arachis hypogaea L. cv. Early Bunch) in a monsoonal tropical environment. Australian Journal of Agricultural Research. 37(4) 361 - 373.

3. Boote, K. J. (1982). Growth Stages of Peanut (Arachis hypogaea L.). Peanut Science. 9(1): 35-40.

4. Caliskan S., M.E. Caliskana, M. Arslan \& H. Arioglu. (2008). Effects of sowing date and growth duration on growth and yield of groundnut in a Mediterranean-type environment in Turkey. Field Crops Research. 105, 131-140.

5. Canavar O. \& M. A. Kaynak. (2010). Effect of Different Planting Dates on Yield and Yield Components of Peanut (Arachis hypogaea L.). Turk J Agric For. 32: 521-528.

6. Chandrika, V., Parameswari, P., \& G. Sreenivas. (2008). Effect of sowing time and rainfall distribution on yield of rainfed groundnut (Arachis hypogea L.) in southern agro-climatic zone of Andhra Pradesh. Legume Research-An International Journal, 31(1), 54-56.

7. Cirilo A. G. \& F. H. Andrade. (1994). Sowing Date and Maize Productivity: I. Crop Growth and Dry Matter Partitioning. Crop Sci. 34:1039-1043.

8. Collino, D. J., Dardanelli, J. L., Sereno, R., \& Racca, R. W. (2001). Physiological responses of argentine peanut varieties to water stress: Light interception, radiation use efficiency and partitioning of assimilates. Field Crops Research, 70 (3), 177-184. 
9. Duncan, W.G., D.E. McCloud, R.L. McGraw, \& K.J. Boote. (1978). Physiological aspects of peanut yield improvement. Crop Sci.18:1015-1020.

10. Gamba, J. M., Grimoldi, A. S., y M. A. Pérez. (2014). Fenología, rendimiento y tamaño de grano de tres variedades comerciales de maní (Arachis hypogaea L.) en condiciones de campo para la zona central de la provincia de Córdoba, Argentina. Agriscientia, 31(1), 25-35.

11. Giambastiani G. (2000). Rendimiento de dos cultivares de maní de distinto ciclo en diferentes épocas de siembra. XV Jornada Nacional de maní. Gral Cabrera - Córdoba. Pag 56.

12. Giayetto O., E.M. Fernandez y G.A. Cerioni. (2006). Fechas y modelos de siembra. En: El Cultivo de maní en Córdoba. Fernandez y Giayetto. Ed. Publicaciones de la Universidad Nacional de Río Cuarto, Río Cuarto, Argentina, 280p.

13. Giayetto O., E.M. Fernandez, G.A. Cerioni, F.D. Morla, M.B. Rosso, M.I.T. Kearney, y M.G. Violante. (2012). Cambios en el rendimiento y la calidad comercial de dos cultivares de maní debido a variaciones de la fecha de siembra, temperatura y radiación. Ciencia y Tecnología de los Cultivos Industriales. 3: 237 - 243.

14. Haro R.H., C. Macedo, L. Gastaldi y C. Casini. (2007a). Efecto de labranza, cultivos antecesores y fechas de siembra sobre la producción y calidad de grano de maní. XXII Jornada Nacional del maní. Gral Cabrera. Córdoba. 20/09/07, pp. 54-56.

15. Haro, R.J., M.E. Otegui, D.J. Collino \& J.L. Dardanelli. (2007b). Environmental effects on seed yield determination of irrigated peanut crops: Links with radiation use efficiency and crop growth rate. Field Crops Research, 103(3): 217-228.

16. Haro, R.J., Baldessari, J. \& Otegui, M.E. (2013). Genetic improvement of peanut in Argentina between 1948 and 2004: Seed yield and its components. Field Crops Research, 149. 76-86.

17. Huyghe, C. (1998). Genetics and genetic modifications of plant architecture in grain legumes: a review. Agronomie, 18(5-6), 383411.

18. Kane, M. V., \& Grabau, L. J. (1992). Early planted, early maturing soybean cropping system: Growth, development, and yield. Agronomy journal, 84(5), 769-773.

19. Kiniry, J.R.; C.E. Simpson, A.M. Schubert \& J.D. Reed. (2005). Peanut leaf area index, light interception, radiation use efficiency, and harvest index at three sites in Texas. Field Crop Research, 91: 297-306. 
20. Kumar, U., Singh, P., \& Boote, K. J. (2012). Effect of Climate Change Factors on Processes of Crop Growth and Development and Yield of Groundnut (Arachis hypogaea L.). Advances in Agronomy, 116, 41-69.

21. Lanier, J. E., Jordan, D. L., Spears, J. F., Wells, R., Johnson, P. D., Barnes, J. S., Hurt, C.A., Brandenburg, R.L. \& J. E. Bailey. (2004). Peanut response to planting pattern, row spacing, and irrigation. Agronomy Journal, 96 (4), 1066-1072.

22. Nanja Reddy, Y. A., Udaya Kumar, M., Prasad, T. G., \& S. C. Aswathnarayana. (1995). Flowering Behaviour and Yield Attributes of Alternate and Sequential Types of Groundnut in Relation to Date of Sowing. Karnataka Journal of Agricultural Sciences, 8(2): 210213.

23. Ong, C. K. (1984). The influence of temperature and water deficits on the partitioning of dry matter in groundnut (Arachis hypogaea L.). Journal of Experimental Botany, 35(5), 746-755.

24. Padmalatha, Y., S. Rami Reddy \& T. Yellamanda Reddy. (2006). The relationship between weather parameters during developmental phase and fruit attributes and yield of peanut. Peanut Science, 33: 118-124.

25. Pedelini R. (2014). Maní. Guía práctica para su cultivo. Boletín de Divulgación Técnica No 2. $3^{\circ}$ Edición. Ediciones INTA. 20 p.

26. Prathima T., T. Yellamanda Reddy, K. M. Dakshina Murthy, T. Murali Krishna, K. Devaki, K. V. Naga Madhuri \& P. Parameswari. (2012). Influence of sowing date and crop variety on phenology, Growth and yield of rainfed groundnut (Arachis hypogaea L.) In Southern zone of Andhra Pradesh, India. International Journal of Applied Biology and Pharmaceutical Technology. 3(1): 26-39. 
Cuadro 1: Duración en días (D) y ${ }^{\circ} \mathrm{Cd}(\mathrm{TT})$ de las etapas de siembra a floración (S-R1), floración a inicio de llenado de grano (R1-R5), llenado de granos (R5-R8) y ciclo total (S-

R8) de los cultivares Utre y Granoleico en función de la fecha de siembra y el ciclo experimental analizado.

\begin{tabular}{|c|c|c|c|c|c|c|c|c|c|c|}
\hline \multirow[t]{3}{*}{ Ciclo } & \multirow{3}{*}{$\begin{array}{c}\text { Fecha } \\
\text { de Siembra }\end{array}$} & \multirow[t]{3}{*}{ Cultivar } & \multicolumn{8}{|c|}{ Duración de la Fenofase } \\
\hline & & & \multicolumn{2}{|c|}{ S-R1 } & \multicolumn{2}{|c|}{ R1-R5 } & \multicolumn{2}{|c|}{ R5-R8 } & \multicolumn{2}{|c|}{ S-R8 } \\
\hline & & & $\mathrm{D}$ & TT & $\mathrm{D}$ & TT & $\mathrm{D}$ & TT & $\mathrm{D}$ & TT \\
\hline \multirow[t]{6}{*}{ 2009/10 } & $1^{\circ}$ & Granoleico & 60 & 578 & 56 & 695 & 45 & 525 & 161 & 1798 \\
\hline & $2^{\circ}$ & Granoleico & 62 & 686 & 49 & 630 & 46 & 429 & 157 & 1745 \\
\hline & $3^{\circ}$ & Granoleico & 42 & 490 & 56 & 703 & $37 *$ & $278 *$ & $135 *$ & $1471^{*}$ \\
\hline & $1^{\circ}$ & Utre & 48 & 315 & 50 & 533 & 50 & 645 & 148 & 1647 \\
\hline & $2^{\circ}$ & Utre & 49 & 536 & 46 & 663 & 51 & 482 & 146 & 1682 \\
\hline & $3^{\circ}$ & Utre & 40 & 462 & 45 & 578 & 48 & 429 & 133 & 1470 \\
\hline \multirow[t]{6}{*}{ 2010/11 } & $1^{\circ}$ & Granoleico & 60 & 498 & 51 & 664 & 60 & 569 & 171 & 1731 \\
\hline & $2^{\circ}$ & Granoleico & 44 & 475 & 49 & 613 & 67 & 580 & 160 & 1668 \\
\hline & $3^{\circ}$ & Granoleico & 42 & 519 & 56 & 609 & $44 *$ & $311^{*}$ & $142 *$ & $1440 *$ \\
\hline & $1^{\circ}$ & Utre & 48 & 378 & 53 & 637 & 56 & 677 & 157 & 1692 \\
\hline & $2^{\circ}$ & Utre & 40 & 413 & 46 & 613 & 59 & 532 & 145 & 1558 \\
\hline & $3^{\circ}$ & Utre & 35 & 455 & 47 & 535 & 59 & 449 & 141 & 1439 \\
\hline
\end{tabular}

* Daño por heladas.

Cuadro 3: Componentes numéricos directos e indirectos del rendimiento de frutos de los cultivares de maní Granoleico y Utre en función de la fecha de siembra en los ciclos experimentales 2009/10 y 2010/11.

\begin{tabular}{|c|c|c|c|c|c|c|}
\hline & $\begin{array}{c}\text { Biomasa } \\
\text { Total } \\
\end{array}$ & IC & $\begin{array}{c}\text { Peso } \\
100 \text { frutos }\end{array}$ & Frutos $\mathrm{m}^{-2}$ & Rendimiento & Confitería \\
\hline & $\mathrm{g} \mathrm{m}^{-2}$ & $\mathrm{~g} \mathrm{~g}^{-1}$ & $\mathrm{~g}$ & \# & $\mathrm{Kg} \mathrm{ha}^{-1}$ & $\%$ \\
\hline & \multicolumn{6}{|c|}{$2009 / 2010$} \\
\hline \multicolumn{7}{|l|}{ Cvar } \\
\hline Granoleico & 1056 & 0,56 & 114 & 574 & 6018,6 & 61,7 \\
\hline Utre-UNRC & 866,5 & 0,55 & 107 & 492 & 4757,2 & 78,5 \\
\hline \multicolumn{7}{|c|}{ Fecha de Siembra } \\
\hline $1^{\circ}$ & 1121,8 & 0,58 & 112 & 589 & 6578,7 & 80 \\
\hline $2^{\circ}$ & 933,9 & 0,58 & 112 & 494 & 5466,3 & 69,8 \\
\hline $3^{\circ}$ & 828,1 & 0,50 & 108 & 517 & 4118,7 & 60,5 \\
\hline \multicolumn{7}{|l|}{ ANAVA* } \\
\hline Cultivar & $\dagger$ & NS & NS & NS & $\dagger$ & $\dagger+\dagger$ \\
\hline Fecha de Siembra & $\dagger$ & $\dagger$ & NS & NS & $\dagger$ & $\dagger \dagger$ \\
\hline \multirow[t]{2}{*}{ Int. Cv x FS } & NS & NS & NS & NS & NS & $\dagger$ \\
\hline & \multicolumn{6}{|c|}{$2010 / 11$} \\
\hline \multicolumn{7}{|l|}{ Cvar } \\
\hline Granoleico & 1261,7 & 0,6 & 109 & 708 & 7703,1 & 68,7 \\
\hline Utre-UNRC & 1092,1 & 0,57 & 100 & 631 & 6243,8 & 75,7 \\
\hline \multicolumn{7}{|c|}{ Fecha de Siembra } \\
\hline $1^{\circ}$ & 1180,2 & 0,62 & 115 & 653 & 7318,7 & 86 \\
\hline $2^{\circ}$ & 1261,7 & 0,62 & 111 & 715 & 7832,8 & 71 \\
\hline $3^{\circ}$ & 1090,3 & 0,53 & 89 & 641 & 5768 & 59,5 \\
\hline \multicolumn{7}{|l|}{ ANAVA* } \\
\hline Cultivar & $\dagger$ & NS & $\dagger$ & $\dagger$ & $\dagger$ & $\dagger+\dagger$ \\
\hline Fecha de Siembra & NS & $\dagger$ & $\dagger \dagger$ & NS & $\dagger \dagger$ & $\dagger+\dagger$ \\
\hline Int. Cv x FS & NS & NS & NS & NS & NS & $\dagger$ \\
\hline
\end{tabular}

$* \dagger \dagger \dagger \mathrm{p}<0,001 ; \dagger \dagger \mathrm{p}<0,01 ; \dagger \mathrm{p}<0,05$; NS no significativo (test LSD de Fisher). En cada columna y ciclo experimental, letras mayúsculas (para fecha de siembra) y minúsculas (para cultivar) indican diferencias estadísticamente significativa 
Cuadro 2. Temperatura media del aire y radiación fotosintéticamente activa incidente (RFAinc) durante las etapas fenológicas de siembra a floración (S-R1), floración a inicio de

llenado de granos (R1-R5), llenado de granos a madurez de cosecha (R5-R8) y ciclo completo (S-R8) de los cultivares de maní (Utre y Granoleico) en las fechas de siembra y los ciclos experimentales analizados.

\begin{tabular}{|c|c|c|c|c|c|c|c|c|}
\hline \multirow{2}{*}{$\begin{array}{l}\text { Cultivar } \\
\text { Fecha de Siembra }\end{array}$} & \multicolumn{4}{|c|}{ Temperatura } & \multicolumn{4}{|c|}{ RFAinc } \\
\hline & S-R1 & R1-R5 & R5-R8 & S-R8 & S-R1 & R1-R5 & R5-R8 & S-R8 \\
\hline & \multicolumn{4}{|c|}{${ }^{\circ} \mathrm{C}$} & \multicolumn{4}{|c|}{$\mathrm{MJ} \mathrm{m}^{-2}$} \\
\hline & \multicolumn{4}{|c|}{$2009 / 10$} & & & & \\
\hline \multicolumn{9}{|l|}{ Granoleico } \\
\hline $1^{\circ}$ & 19,9 & 22,6 & 22,0 & 21,5 & 732 & 716 & 445 & 1893 \\
\hline $2^{\circ}$ & 21,2 & 23,2 & 19,7 & 21,4 & 727 & 593 & 439 & 1759 \\
\hline $3^{\circ}$ & 22,0 & 22,9 & 17,8 & 21,4 & 520 & 603 & 321 & 1445 \\
\hline \multicolumn{9}{|l|}{ Utre } \\
\hline $1^{\circ}$ & 20,0 & 20,9 & 23,2 & 21,4 & 598 & 587 & 574 & 1759 \\
\hline $2^{\circ}$ & 21,2 & 23,6 & 20,9 & 21,9 & 577 & 605 & 485 & 1667 \\
\hline \multirow[t]{2}{*}{$3^{\circ}$} & 21,9 & 21,2 & 19,2 & 21,4 & 488 & 509 & 435 & 1433 \\
\hline & \multirow{2}{*}{\multicolumn{4}{|c|}{$2010 / 11$}} & & & & \\
\hline \multicolumn{5}{|l|}{ Granoleico } & & & & \\
\hline $1^{\circ}$ & 18,5 & 23,3 & 19,8 & 20,4 & 734 & 749 & 694 & 2177 \\
\hline $2^{\circ}$ & 20,8 & 22,8 & 18,9 & 20,7 & 593 & 690 & 710 & 1995 \\
\hline $3^{\circ}$ & 23,1 & 20,8 & 17,0 & 20,3 & 611 & 689 & 401 & 1701 \\
\hline \multicolumn{9}{|l|}{ Utre } \\
\hline $1^{\circ}$ & 18,0 & 22,3 & 21,3 & 20,6 & 588 & 741 & 688 & 2017 \\
\hline $2^{\circ}$ & 20,4 & 23,6 & 19,3 & 21,0 & 528 & 675 & 655 & 1859 \\
\hline $3^{\circ}$ & 22,9 & 21,7 & 17,9 & 20,4 & 499 & 616 & 583 & 1698 \\
\hline
\end{tabular}

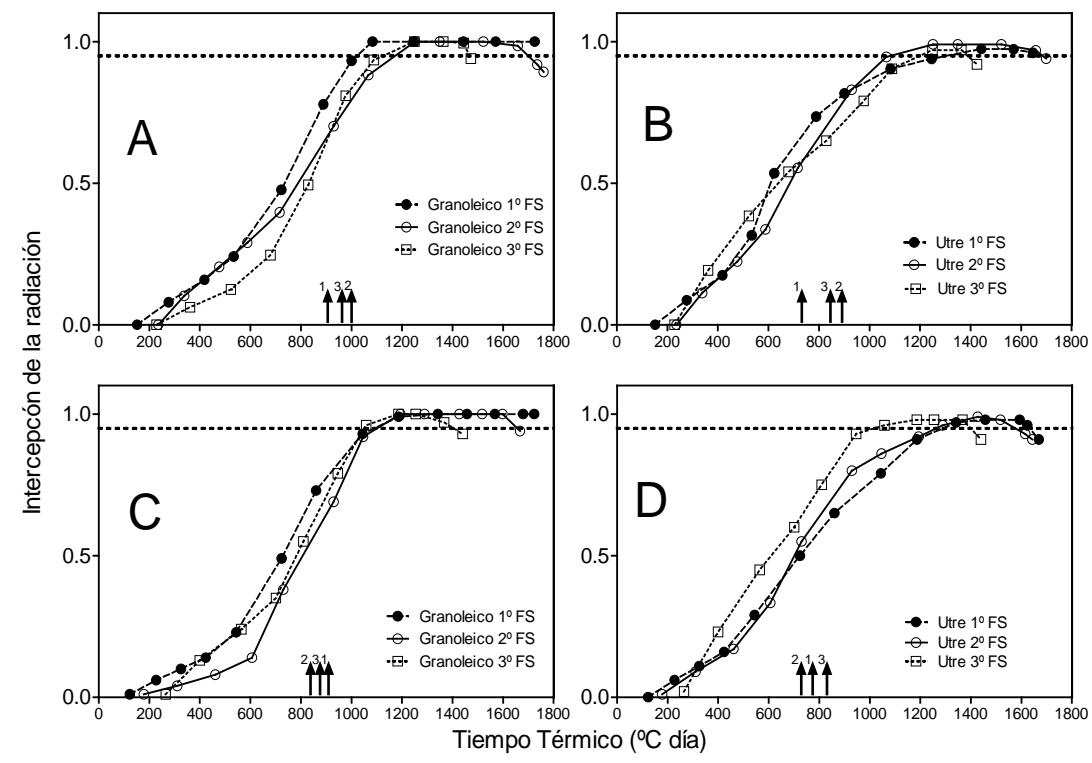

Figura 1: Intercepción de la radiación fotosintéticamente activa incidente en función del tiempo térmico después de la emergencia para los cultivares Granoleico (A y C) y Utre (B y D), sembrados en diferentes fechas de siembra durante los ciclos 2009/10 (A y B) y 2010/11

(C y D). Las flechas indican inicio del periodo crítico, comienzo de formación de frutos (R3), para cada fecha de siembra, indicado como 1, 2 y 3. 

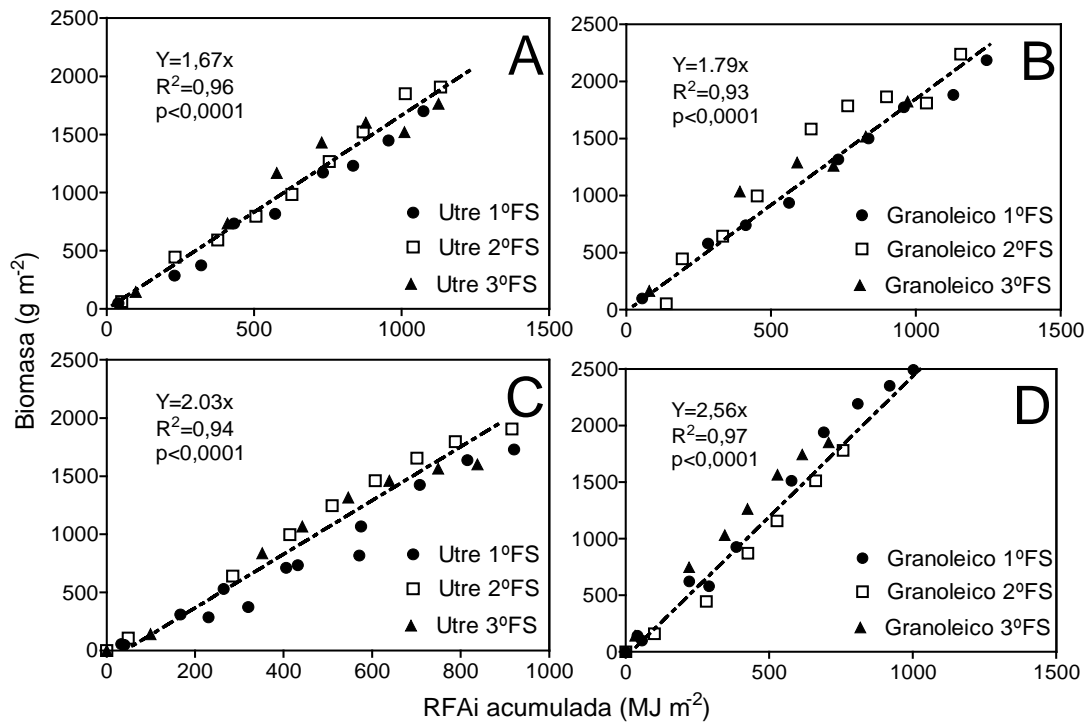

Figura 2: Relación entre la biomasa aérea acumulada y la RFA interceptada a lo largo del ciclo de los cultivares Utre (A y C) y Granoleico (B y D) para las campañas agrícolas 2009/10 (A y B) y 2010/11 (C y D). La pendiente de la función lineal representa la eficiencia de uso de la radiación (EUR) fotosintéticamente activa interceptada.
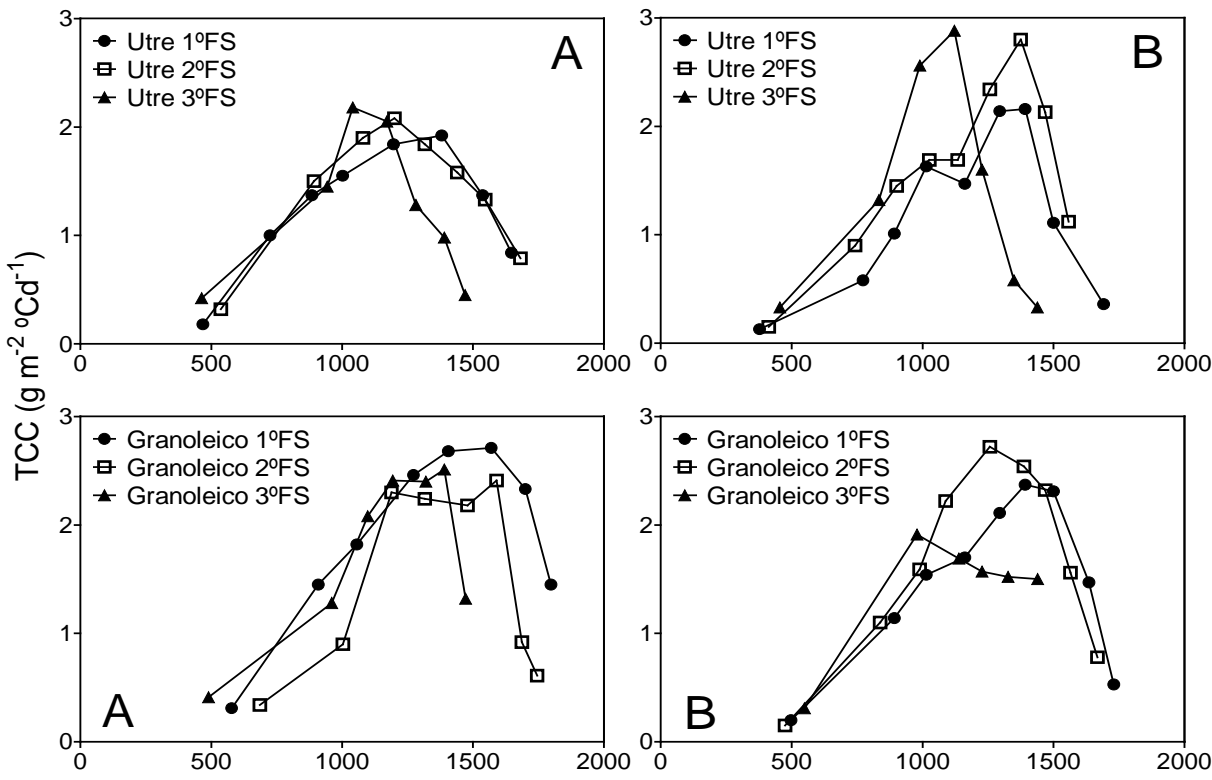

Tiempo Térmico $\left({ }^{\circ} \mathrm{C}\right.$ día)

Figura 3: Dinámica temporal de la tasa de crecimiento del cultivo (TCC) del cultivar Utre y Granoleico en función del tiempo térmico después de la siembra $\left({ }^{\circ} \mathrm{Cd}\right)$, para las tres fechas de siembra y ambos ciclos experimentales 2009/10 (A) y 2010/11 (B). 


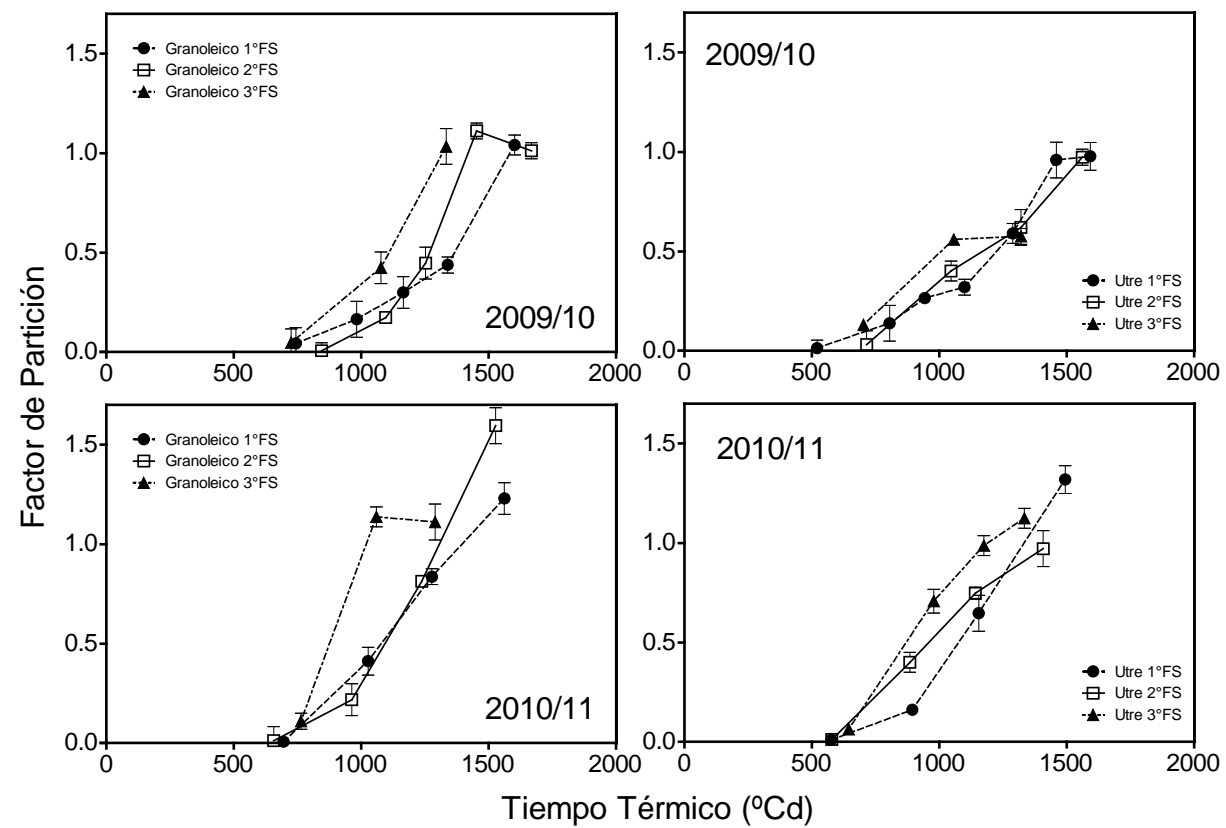

Figura 4: Factor de partición de materia seca a frutos de los cultivares Granoleico y Utre en función del tiempo térmico ( ${ }^{\circ} \mathrm{C}$ día) desde la siembra, según fecha de siembra y ciclo experimental. 